\title{
Identification of nonlinear block-oriented systems with backlash and saturation
}

\author{
Jozef Vörös*
}

\begin{abstract}
A new approach to modeling and identification of discrete-time nonlinear dynamic systems with input backlash and output saturation nonlinearities is presented. The proposed three-block cascade mathematical model results from successive applications of the key-term separation principle. This provides special nonlinear model description that is linear in parameters. An iterative technique with internal variable estimation is proposed for estimation of all the model parameters based on measured input/output data and minimizing the least-squares criterion. Illustrative example of cascade system identification with backlash and saturation is included.
\end{abstract}

K e y w o r d s: nonlinear systems, cascade models, identification, backlash, saturation

\section{Introduction}

The system identification is always based on classes of appropriate mathematical models. By definition, the system identification is the determination, on the basis of observations of input and output, of a model within a specified class of models to which the system is equivalent in terms of a criterion. It means that given a class of mathematical models, the system identification involves finding one specific model, which may be regarded as equivalent to the real system with respect to input-output data.

In contrast to linear dynamic systems, there is no universal mathematical model for characterization or description of nonlinear dynamic systems; therefore, specific models have to be considered in modeling and identification of different types of real nonlinear control systems. The main problem in modeling of nonlinear dynamic systems is the variety of system structures and the types of real or assumed nonlinear characteristics. In choosing of an appropriate class of nonlinear mathematical models, both above-mentioned aspects have to be considered and very often classes of particular models have to be constructed for different types of real systems.

One way to specify nonlinear dynamic model structure is to combine linear dynamic submodels with nonlinear static (memoryless) submodels or blocks creating the so-called block-oriented models. Commonly used blockoriented models are the so-called Hammerstein model, Wiener model and their combinations. The Hammerstein model consists of a nonlinear static block followed by a linear dynamic block and many researchers have studied the identification of nonlinear dynamic systems using Hammerstein model. The identification techniques mainly distinguish themselves in the way the static nonlinearity is represented and in the form of optimization problem that is finally obtained. Known approaches for different types of nonlinearities and estimation methods can be found $e g$ in [1-13]. The Wiener model has a linear dynamic block followed by a nonlinear static block and many techniques have been proposed to solve the identification problems typically encountered in using this model, see, eg [14-26].

More complex nonlinear dynamic systems can be modeled by putting more block-oriented models in series. One of them is the HammersteinWiener model that consists of a linear dynamic block embedded between two static nonlinear blocks. In control systems, the use of this model is motivated by considering the input nonlinear block as the actuator nonlinearity and the output nonlinear block as the process nonlinearity or sensor nonlinearity. More identification approaches are using this model; see eg [27-33]. Note that the nonlinear blocks of Hammerstein model, Wiener model and their combinations are assumed to be static, ie without memory.

The type of nonlinearity significantly influences the form of mathematical model. This is evident in the case of nonsmooth dynamic nonlinearities, where a multi-valued mapping characterizes the behavior of nonlinearity. As none of the above-mentioned block-oriented models can be used if nonsmooth dynamic nonlinearities exist in control systems, special block-oriented models are required.

* Slovak University of Technology, Faculty of Electrical Engineering and Information Technology, Ilkovičova 3 , 81219 Bratislava, Slovakia, jozef.voros@stuba.sk 
Backlash is one of the most important nonlinearities that can be classified as dynamic (ie with memory) and hard (non-differentiable). This nonlinearity commonly occurs in magnetic, hydraulic and mechanical components, eg bearings, gears [34]. The ignorance of backlash will worsen the system performance and even lead to serious instability; therefore, the identification of systems with backlash is of great importance. More approaches were presented on identification of two-block cascade systems with input backlash followed by a linear dynamic subsystem [35-43].

In this paper, an approach to the parameter identification of nonlinear dynamic systems using three-block cascade models is presented where a nonlinear dynamic block with backlash is followed by a linear dynamic block and this is followed by a nonlinear static block with saturation, ie in this case the position of nonlinear blocks significantly differs from the model structure in [44] and also from [45] because of output saturation. Repeated application of the key-term separation principle enables to separate the parameters of the model blocks and to reduce the redundant parameters. This improves the calculation efficiency. An iterative technique with internal variable estimation is proposed for estimation of all the model parameters based on measured input/output data and minimizing the least-squares criterion. Illustrative example of cascade systems identification with input backlash and output saturation characteristics is included.

\section{MATHEMATICAL MODEL DESCRIPTION}

Let the nonlinear dynamic system be modeled by the serial connection of a nonlinear dynamic block with backlash followed by a linear dynamic block, which is followed by a nonlinear static block. The input backlash characteristic with input $u(t)$ and output $v(t)$ is described by two straight lines, upward (right) and downward (left) sides of backlash, connected with horizontal line segments [35]. The backlash behavior for the discrete-time case is given by

$$
v(t)= \begin{cases}m_{L}\left[u(t)+c_{L}\right], & u(t) \leq z_{L}, \\ m_{R}\left[u(t)-c_{R}\right], & u(t) \geq z_{R} \\ v(t-1), & z_{L} \leq u(t) \leq z_{R}\end{cases}
$$

for $t=1,2,3, \ldots$ where $m_{L}$ is the slope of left line of backlash and $c_{L}$ is the distance between its intersection with $\mathrm{u}$-axis and the origin (left dead zone), while $m_{\mathrm{R}}$ is the slope of right line of backlash and $c_{\mathrm{R}}$ is the distance between its intersection with $u$-axis and the origin (right dead zone). It means that the backlash is characterized by 4 constant parameters and by

$$
\begin{aligned}
& z_{L}=\frac{v(t-1)}{m_{L}}-c_{L}, \\
& z_{R}=\frac{v(t-1)}{m_{R}}+c_{R},
\end{aligned}
$$

$i e$ the $u$-axis values of intersections of the two lines, with slopes $m_{L}, m_{R}$, with the horizontal inner segment containing $v(t-1)$. The slopes of straight lines $m_{L}$ and $m_{R}$ may be simultaneously positive or negative, while $c_{L}$ and $c_{R}$ must be positive. This model allows the upward and downward line slopes to be different if the intersection of the two lines is not in the region of practical interest.

The backlash model can be described by the following first-order difference equation [42]

$$
\begin{array}{r}
v(t)=m_{L} u(t) f_{1}(t)+m_{L} c_{L} f_{1}(t)+m_{R} u(t) f_{2}(t)- \\
m_{R} c_{R} f_{2}(t)+v(t-1)\left[1-f_{1}(t)\right]\left[1-f_{2}(t)\right]
\end{array}
$$

where

$$
\begin{aligned}
f_{1}(t) & =h\left[u(t)-z_{L}\right] \\
& =h\left\{\left[m_{L} u(t)+m_{L} c_{L}-v(t-1)\right] / m_{L}\right\}, \\
f_{2}(t) & =h\left[z_{R}-u(t)\right] \\
& =h\left\{\left[v(t-1)-m_{R} u(t)+m_{R} c_{R}\right] / m_{R}\right\}
\end{aligned}
$$

are internal variables based on (2) and (3) using the following function

$$
h(\sigma)= \begin{cases}0, & \text { if } \sigma>0 \\ 1, & \text { if } \sigma \leq 0\end{cases}
$$

switching between two sets of values, ie, $(-\infty, \sigma)$ and $(\sigma, \infty)$. The input/output relation (4) is identical with that of (1).

The linear dynamic block of the three-block cascade model can be described by the following input/output relation

$$
x(t)=A\left(q^{-1}\right) v(t)+\left[1-B\left(q^{-1}\right)\right] x(t)
$$

where $v(t)$ and $x(t)$ are the inputs and outputs, respectively, $A\left(q^{-1}\right)$ and $B\left(q^{-1}\right)$ are two coprime polynomials in the unit delay operator $q^{-1}\left(\right.$ ie $\left.q^{-1} x(t)=x(t-1)\right)$

$$
\begin{aligned}
& A\left(q^{-1}\right)=a_{1} q^{-1}+\ldots+a_{r} q^{-r}, \\
& B\left(q^{-1}\right)=1+b_{1} q^{-1}+\ldots+b_{p} q^{-p}
\end{aligned}
$$

Finally assume that the output $y(t)$ of nonlinear static block can be described by the $(2+2)$-segment form of general multisegment piecewise linear function [25] given as follows

$$
\begin{aligned}
y(t)= & m_{R 1} x(t)+\left(m_{L 1}-m_{R 1}\right) h[x(t)] x(t) \\
& +\left(m_{R 2}-m_{R 1}\right) h\left[D_{R}-x(t)\right] x(t)-D_{R} g_{1}(t) \\
& +\left(m_{L 2}-m_{L 1}\right) h\left[x(t)-D_{L}\right] x(t)-D_{L} g_{2}(t)
\end{aligned}
$$

with the following internal variables

$$
\begin{aligned}
& g_{1}(t)=\left(m_{R 2}-m_{R 1}\right) h\left[D_{R}-x(t)\right], \\
& g_{2}(t)=\left(m_{L 2}-m_{L 1}\right) h\left[x(t)-D_{L}\right]
\end{aligned}
$$


where $\left|m_{R 1}\right|<\infty,\left|m_{R 2}\right|<\infty$ are the linear segment slopes and $0<D_{R}<\infty$ is the constant for the positive inputs, $\left|m_{L 1}\right|<\infty,\left|m_{L 2}\right|<\infty$ are the linear segment slopes and $-\infty<D_{L} \leq 0$ is the constant for the negative inputs and $h(\cdot)$ is the above-defined switching function. In the case of saturation, the slopes $m_{R 2}$ and $m_{L 2}$ are equal to zero.

The three-block cascade model description can be created by consecutive substitutions of (4) into (8) and then the result of this substitution into (11). However, this will result in a very complex equation with crossmultiplications of parameters. Therefore, the so-called key-term separation principle $[10,25]$ will be applied to transform the system described by (4), (8) and (11) into a pseudo-linear representation without the products of parameters. Moreover, the parameterization of the cascade connection of three blocks is not unique, as many combinations of block parameters can be found. To get a unique parameterization, one parameter has to be fixed in at least two blocks. For application of the key term separation principle, it is appropriate to choose $a_{1}=1$ in (8), and $m_{R 1}=1$ in (11).

To construct the input/output equation of the cascade model, we separate the first term in the description the linear dynamic block as follows

$$
x(t)=v(t-1)+\sum_{i=2}^{r} a_{i} v(t-i)-\sum_{j=1}^{p} b_{j} x(t-j)
$$

and then we half-substitute (4) into (14), ie only for the separated term

$$
\begin{aligned}
x(t)=m_{L} u(t-1) & f_{1}(t-1)+m_{L} c_{L} f_{1}(t-1) \\
+m_{R} u(t-1) f_{2}(t-1)-m_{R} c_{R} f_{2}(t-1) & \\
+v(t-2)[ & {\left[1-f_{1}(t-1)\right]\left[1-f_{2}(t-1)\right] } \\
& +\sum_{i=2}^{r} a_{i} v(t-i)-\sum_{j=1}^{p} b_{j} x(t-j) .
\end{aligned}
$$

Finally, the output equation of this cascade model can be constructed by half-substituting (15) into (11), ie only for the first term in the description of output block

$$
\begin{aligned}
& y(t)=m_{1} u(t-1) f_{1}(t-1)+c_{1} f_{1}(t-1) \\
&+ m_{2} u(t-1) f_{2}(t-1)-c_{2} f_{2}(t-1) \\
&+ v(t-2)\left[1-f_{1}(t-1)\right]\left[1-f_{2}(t-1)\right] \\
& \quad+\sum_{i=2}^{r} a_{i} v(t-i)-\sum_{j=1}^{p} b_{j} x(t-j) \\
&+M h[x(t)] x(t)+M_{1} h\left[D_{R}-x(t)\right] x(t)-D_{R} g_{1}(t) \\
&+M_{2} h\left[x(t)-D_{L}\right] x(t)-D_{L} g_{2}(t)+e(t)
\end{aligned}
$$

with

$$
\begin{aligned}
m_{L} & =m_{1}, c_{L}=c_{1} / m_{1}, m_{R}=m_{2}, c_{R}=c_{2} / m_{2}, \\
M & =m_{L, 1}-1, \\
M_{1} & =m_{R 2}-m_{R 1}, M_{2}=m_{L 2}-m_{L 1},
\end{aligned}
$$

where the assumed measurement noise $e(t)$ is a white noise with zero mean. Now the parameters of the input backlash, the linear dynamic block and the output nonlinearity are separated and the equation is quasi-linear as the internal variables $f_{1}(t)$ and $f_{2}(t)$ depend on the backlash parameters while $g_{1, i}(t)$ and $g_{2, j}(t)$ depend on the parameters of output nonlinearity.

\section{PARAMETER ESTIMATION}

Assume that the linear dynamic subsystem is stable, the degrees of polynomials $A\left(q^{-1}\right)$ and $B\left(q^{-1}\right)$ and the numbers of piecewise linear function segments are known and $y(t)=0, u(t)=0, x(t)=0$ and $v(t)=0$ for $t \leq 0$. The input/output equation (16) can be used for estimation of all the model parameters. Defining the following vector of data

$$
\begin{gathered}
\phi(t)=\left[u(t-1) f_{1}(t-1), f_{1}(t-1), u(t-1) f_{2}(t-1),\right. \\
-f_{2}(t-1), v(t-2), \ldots, v(t-r),-x(t-1), \ldots,-x(t-p), \\
h[x(t)] x(t), h\left[D_{R}-x(t)\right] x(t),-g_{1}(t), \\
\left.h\left[x(t)-D_{L}\right] x(t),-g_{2}(t)\right]^{\top}
\end{gathered}
$$

and the parameter vector

$$
\begin{array}{r}
\theta=\left[m_{1}, c_{1}, m_{2}, c_{2}, a_{2}, \ldots, a_{r}, b_{1}, \ldots, b_{p},\right. \\
\left.M, M_{1}, D_{R}, M_{2}, D_{L}\right]^{\top},
\end{array}
$$

the three-block cascade model can be written in the following form

$$
\begin{array}{r}
y_{c}(t)=y(t)-v(t-2)\left[1-f_{1}(t-1)\right]\left[1-f_{2}(t-1)\right] \\
=\phi^{\top}(t) \theta+e(t) .
\end{array}
$$

Considering $N$ observations of inputs $u(t)$ and outputs $y(t)$ and assuming that $u(t)=0$ and $y(t)=0$ for $t \leq 0$, we can define the stacked corrected output vector $Y_{c}(N)$, the stacked information matrix $\Phi(N)$ and the stacked noise vector $E(N)$ as

$$
\begin{aligned}
Y_{c}(N) & =\left[y_{c}(1), y_{c}(2), \ldots, y_{c}(N)\right]^{\top}, \\
\Phi(N) & =[\phi(1), \phi(2), \ldots, \phi(N)]^{\top} \\
E(N) & =[e(1), e(2), \ldots, e(N)]^{\top},
\end{aligned}
$$

then from (20), we have

$$
Y_{c}(N)=\Phi(N) \theta+E(N) .
$$

Minimizing the least-squares criterion function

$$
J(\theta)=\left\|Y_{c}(N)-\Phi(N) \theta\right\|^{2}
$$


with respect to $\theta$ and assuming that the information matrix $\Phi(N)$ is persistently exciting, ie $\Phi^{\top}(N) \Phi(N)$ is an invertible matrix, we can obtain the least squares estimate of the parameter vector as

$$
\hat{\theta}=\left[\Phi^{\top}(N) \Phi(N)\right]^{-1} \Phi^{\top}(N) Y_{c}(N)
$$

However, the information matrix $\Phi(N)$ and the corrected output vector $Y_{c}(N)$ contain unknown internal variables, therefore an iterative algorithm must be applied for estimation of model parameters. The technique presented in [42], which is based on the use of the preceding estimates of model parameters for the estimation of internal variables and vice-versa, can be easily extended to this three-block model. We replace the internal variables and the corrected output in $s$-th iteration by their estimates

$$
\begin{gathered}
{ }^{s} v(t)={ }^{s} m_{1} u(t){ }^{s} f_{1}(t)+{ }^{s} c_{1}{ }^{s} f_{1}(t)+{ }^{s} m_{2} u(t){ }^{s} f_{2}(t) \\
-{ }^{s} c_{2}{ }^{s} f_{2}(t)+{ }^{s} v(t-1)\left[1-{ }^{s} f_{1}(t)\right]\left[1-{ }^{s} f_{2}(t)\right], \\
{ }^{s} m_{L}={ }^{s} m_{1},{ }^{s} c_{L}={ }^{s} c_{1} /{ }^{s} m_{1},{ }^{s} m_{R}={ }^{s} m_{2},{ }^{s} c_{R}={ }^{s} c_{2} /{ }^{s} m_{2}, \\
{ }^{s} f_{1}(t)=h\left\{\left[{ }^{s} m_{L} u(t)+{ }^{s} m_{L}{ }^{s} c_{L}-{ }^{s} v(t-1)\right] /{ }^{s} m_{L}\right\}, \\
{ }^{s} f_{2}(t)=h\left\{\left[{ }^{s} v(t-1)-{ }^{s} m_{R} u(t)+{ }^{s} m_{R}{ }^{s} c_{R}\right] /{ }^{s} m_{R}\right\} \\
{ }^{s} x(t)={ }^{s} v(t-1)+\sum^{r}{ }^{s} a_{i}{ }^{s} v(t-i)-\sum^{p}{ }^{s} b_{j}{ }^{s} x(t-j) \\
{ }^{s} g_{1}(t)={ }^{s} M_{1} h\left[{ }^{s} D_{R}-{ }^{s} x(t)\right], \\
{ }^{s} g_{2}(t)={ }^{s} M_{2} h\left[{ }^{s} x(t)-{ }^{s} D_{L}\right],
\end{gathered}
$$

and defining the estimate of the data vector

${ }^{s} \phi(t)=\left[u(t-1)^{s} f_{1}(t-1),{ }^{s} f_{1}(t-1), u(t-1)^{s} f_{2}(t-1)\right.$,

$-{ }^{s} f_{2}(t-1),{ }^{s} v(t-2), \ldots,{ }^{s} v(t-r),-{ }^{s} x(t-1), \ldots,-{ }^{s} x(t-p)$,

$$
\begin{array}{r}
h\left[{ }^{s} x(t)\right]{ }^{s} x(t), h\left[{ }^{s} D_{R}-{ }^{s} x(t)\right]{ }^{s} x(t),-{ }^{s} g_{1}(t), \\
\left.h\left[{ }^{s} x(t)-{ }^{s} D_{L}\right]{ }^{s} x(t),-{ }^{s} g_{2}(t)\right]{ }^{\top}
\end{array}
$$

and forming the corrected output vector ${ }^{s} Y_{c}(N)$ and the information matrix ${ }^{s} \Phi(N)$

$$
\begin{aligned}
{ }^{s} Y_{c}(N) & =\left[{ }^{s} y_{c}(1),{ }^{s} y_{c}(2), \ldots,{ }^{s} y_{c}(N)\right]^{\top}, \\
{ }^{s} \Phi(N) & =\left[{ }^{s} \phi(1),{ }^{s} \phi(2), \ldots,{ }^{s} \phi(N)\right]^{\top},
\end{aligned}
$$

we will minimize the least-squares criterion function

$$
J\left({ }^{s+1} \theta\right)=\left\|{ }^{s} Y_{c}(N)-{ }^{s} \Phi(N){ }^{s+1} \theta\right\|^{2}
$$

with respect to ${ }^{s+1} \theta$ giving

$$
{ }^{s+1} \theta=\left[{ }^{s} \Phi^{\top}(N){ }^{s} \Phi(N)\right]^{-1} s \Phi^{\top}(N){ }^{s} Y_{c}(N)
$$

If the input $u(t)$ is persistently excited with respect to the dead zone of input backlash and uncorrelated with the output noise $e(t)$, the steps of the iterative procedure for the given set of input/output data $\{u(t) ; y(t)\}, t=$ $1,2, \ldots, N$, are listed as follows:

1) For $s=1$, set ${ }^{1} v(t)=u(t)$ and ${ }^{1} x(t)=y(t)$, consider nonzero initial values of backlash parameters $\left(e{ }^{1} m_{L}={ }^{1} m_{R}=1\right.$ for the backlash with positive slopes or ${ }^{1} m_{L}={ }^{1} m_{R}=-1$ for the backlash with negative slopes, while ${ }^{1} c_{L}$ and ${ }^{1} c_{R}$ are chosen small enough).

2) Compute the estimates of internal variables using $(27)-(33)$.

3) Compute the estimates of corrected outputs using (34).

4) Form the corrected output vector ${ }^{s} Y_{c}(N)$ and the information matrix ${ }^{s} \Phi(N)$.

5) Update the parameter estimates ${ }^{s+1} \theta$ using (39).

6 ) For some pre-set small $\delta$, if $J\left({ }^{s+1} \theta\right)<\delta$, then the iterative procedure terminates otherwise, put $s=s+1$ and go to Step 2.

Note, that only the linear dynamic block and output block parameters are estimated in the first iteration, if we choose very small ${ }^{1} c_{L}$ and ${ }^{1} c_{R}$.

\section{SIMULATION STUDIES}

The proposed identification method for the identification of systems with backlash input and multisegment piecewise linear output nonlinearities was implemented and tested in MATLAB. Several cases were simulated and the estimations of parameters were carried out on the basis of input and output records. The performance of the proposed methods is illustrated on the following example.

EXAMPLE. The cascade system with an input backlash (Fig. 1) characterized by the parameters $m_{L}=0.8, c_{L}=$ $0.3, m_{R}=0.9, c_{R}=0.2$, followed by the linear dynamic system described by the difference equation

$x(t)=v(t-1)+0.5 v(t-2)+0.2 x(t-1)-0.35 x(t-2)$

and the following saturation (Fig. 2) given by

$$
y(t)= \begin{cases}x(t) & \text { if } 0 \leq x(t) \leq 0.9 \\ 0.9 & \text { if } x(t)>0.9 \\ 0.6 x(t) & \text { if }-0.8 \leq x(t)<0 \\ -0.48 & \text { if } x(t)<-0.8\end{cases}
$$

(ie $M=-0.4, M_{1}=-1.0, M_{2}=-0.6, D_{R}=0.9$ and $\left.D_{L}=-0.8\right)$ was simulated using (16). 


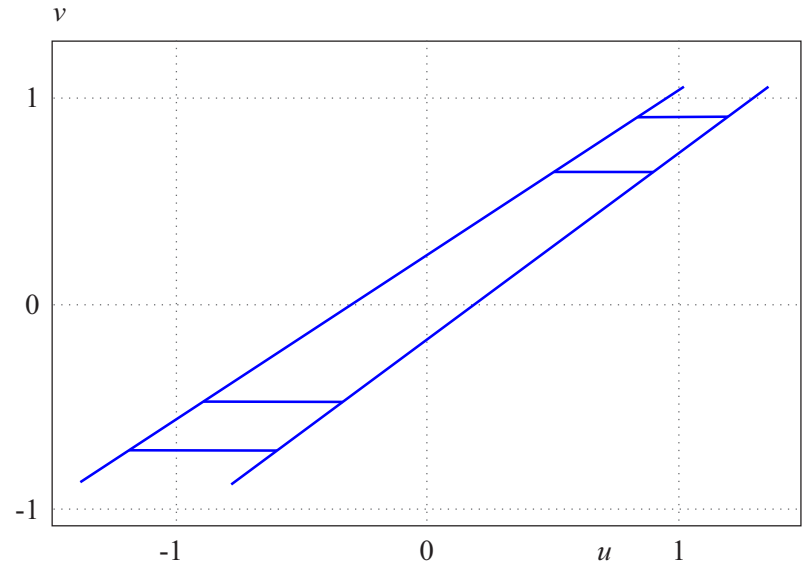

Fig. 1. Input backlash

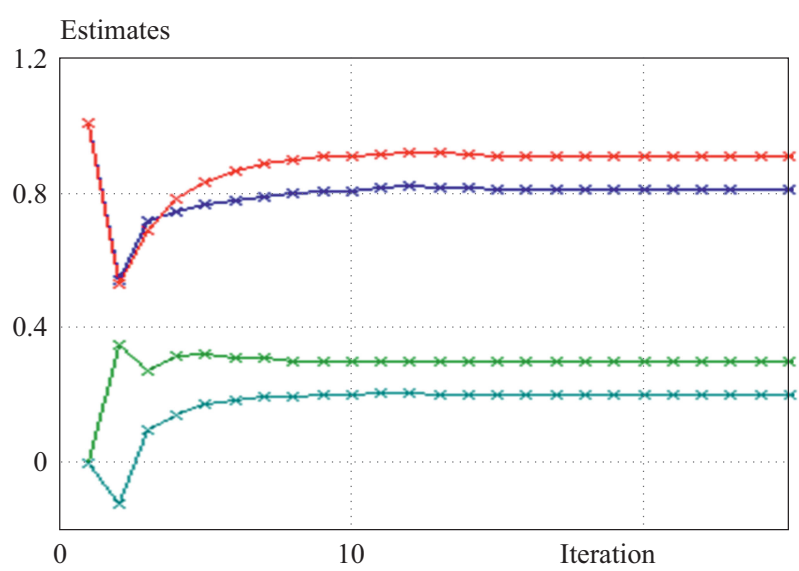

Fig. 3. The process of parameter estimation for the backlash

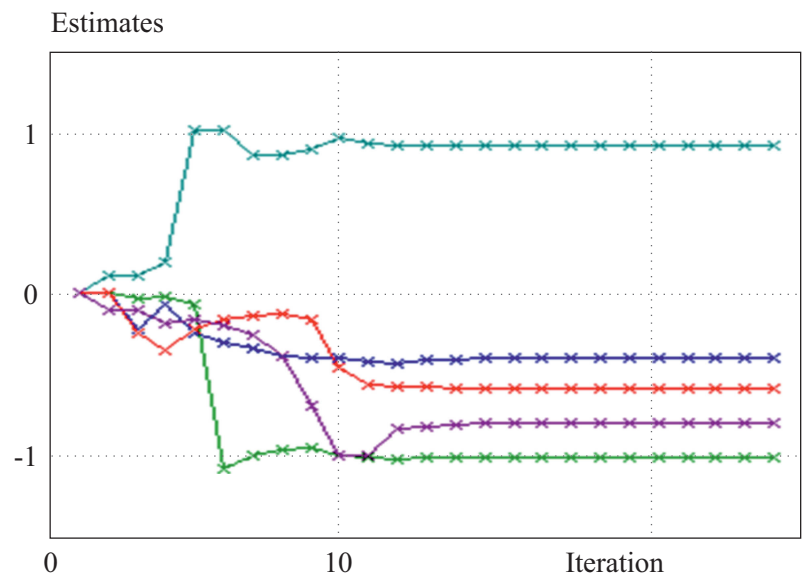

Fig. 5. The process of parameter estimation for the nonlinear block

The identification was performed on the basis of 3000 samples of uniformly distributed random inputs with $|u(t)|<1.0$ and simulated outputs. Normally distributed random noise with zero mean and signal-to-noise ratio $\mathrm{SNR}=25$ (SNR is the square root of the ratio of output and noise variances) was added to the outputs. The iterative estimation algorithm was applied with initial values $m_{L}=m_{R}=1$ and $c_{L}=c_{R}=0.001$ for the first esti-

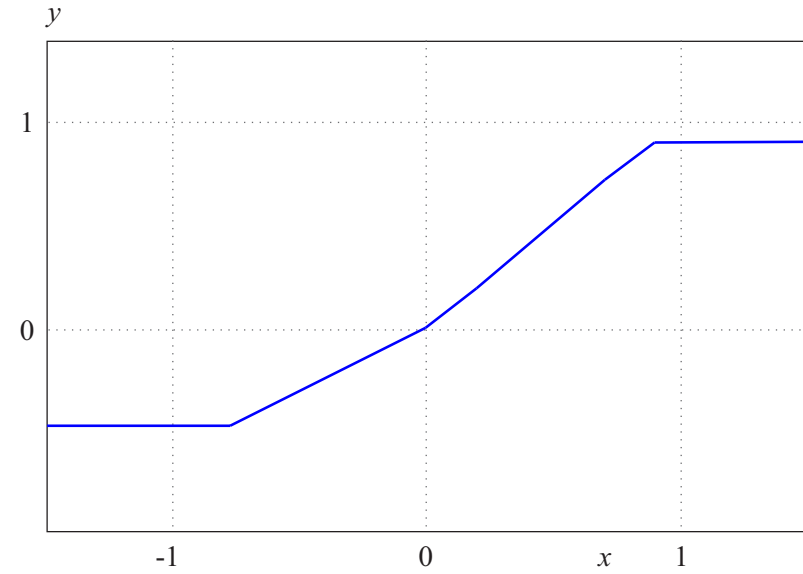

Fig. 2. Output saturation

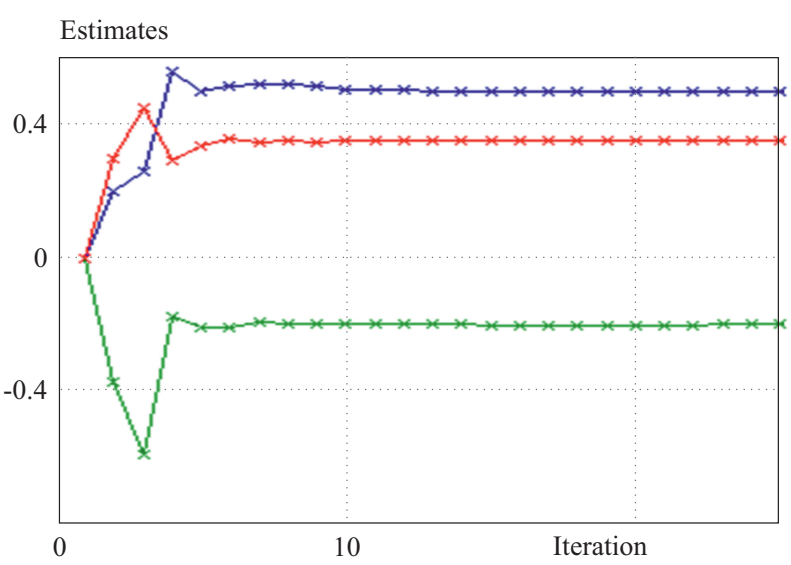

Fig. 4. The process of parameter estimation for the linear dynamic block

mate of $f_{1}(t)$ and $f_{2}(t)$, while the initial values of linear system parameters were chosen zero.

The process of parameter estimation is shown in Fig. 3 for the backlash (the top-down order of parameters is $\left.m_{R}, m_{L}, c_{L}, c_{R}\right)$, in Fig. 4 for the linear block (the top-down order of parameters is $\left.a_{2}, b_{2}, b_{1}\right)$ and in Fig. 5 for the saturation (the top-down order of parameters is $\left.D_{R}, M, M_{2}, D_{L}, M_{1}\right)$. The estimates meet the values of real parameters after about 15 iterations.

It is worth mentioning that although there exist proofs of convergence for iterative identification algorithms considering the cascade of nonlinear static, linear dynamic and nonlinear static blocks, unfortunately no proof of convergence exists for the presented cascade model. However, testing of the proposed algorithm and the above example show that the convergence rate is relatively high despite the complex structure of proposed model with the backlash, as a multivalued mapping, and the additive output noise.

\section{CONCLUSION}

The paper deals with modeling and identification of nonlinear cascade systems with input backlash and out- 
put saturation nonlinearities. The presented iterative parameter estimation approach is based on the three-block cascade model that is linear in parameters. The output block description is appropriate also for the identification of systems with piecewise linear output nonlinearities.

As the proposed nonlinear mathematical model is linear in parameters it can be directly applied in on-line identification of nonlinear dynamic systems with input backlash and output saturation using the known recursive least-squares algorithm [46,47]. Moreover, the presented identification method can be easily extended for systems with the so-called general input backlash [48-51] and also other types of static nonlinearities can be considered in the output block.

\section{Acknowledgements}

The author gratefully acknowledges financial support from the Slovak Scientific Grant Agency (VEGA).

\section{REFERENCES}

[1] H. Chen, F. Ding, and Y. Xiao, "Decomposition-based Least Squares Parameter Estimation Algorithm for Input Nonlinear Systems using the Key Term Separation Technique", Nonlinear Dynamics vol. 79, no. 3, 2015, pp. 2027-2035.

[2] K. Deng and F. Ding, "Newton Iterative Identification Method for an Input Nonlinear Finite Impulse Response System with Moving Average Noise using the Key Variables Separation Technique", Nonlinear Dynamics vol. 76, no. 2, 2014, pp. 1195-1202.

[3] F. Ding, F. Wang, L. Xu, and M. Wu, "Decomposition based Least Squares Iterative Identification Algorithm for Multivariate Pseudo-Linear ARMA Systems using the Data Filtering", Journal of the Franklin Institute vol. 354, no. 3, 2017, pp. 1321-1339.

[4] G. Mzyk, "Instrumental Variables for Nonlinearity Recovering in Block-Oriented Systems Driven by Correlated Signals", International Journal of Systems Science vol. 46, no. 2, 2015, pp. 246-254.

[5] J. Li, F. Ding, and L. Hua, "Maximum likelihood Newton recursive the Newton iterative estimation algorithms for Hammerstein CARAR systems", Nonlinear Dynamics vol. 75, no 1-2, 2014, pp. 235-245.

[6] J. Ma and F. Ding, "Filtering-Based Multistage Recursive Identification Algorithm for an Input Nonlinear Output-Error Autoregressive System by Using the Key Term Separation Technique", Circuits Systems, Signal Processing vol. 36, no. 2, 2017, pp. 577-599.

[7] J. Ma, W. Xiong, F. Ding, A. Alsaedi, and T. Hayat, "Data Filtering based Forgetting Factor Stochastic Gradient Algorithm for Hammerstein Systems with Saturation Preload Nonlinearities", Journal of the Franklin Institute vol. 353, no. 16, 2016, pp. 4280-4299.

[8] V. Saini and L. Dewan, "Instrument Variable Method Based on Nonlinear Transformed Instruments for Hammerstein System Identification", Journal of Vibration Control vol. 24, no 13, 2018, pp. 2802-2814.

[9] Q. Shen and F. Ding, "Hierarchical Multi-Innovation Extended Stochastic Gradient Algorithms for Input Nonlinear Multivariable OEMA Systems by the Key-Term Separation Principle", Nonlinear Dynamics vol. 85, no 1, 2016, pp. 499-507.

[10] J. Vörös, "Iterative Algorithm for Parameter Identification of Hammerstein Systems with Two-Segment Nonlinearities", IEEE Trans. Automatic Control vol. 44, no 11, 1999, pp. 2145-2149.

[11] X. Wang, F. Ding, T. Hayat, and A. Alsaedi, "Combined State Multi-Innovation Parameter Estimation for an Input Non-Linear
State-Space System using the Key Term Separation", IET Control Theory \& Applications vol. 10, no 13, 2016, pp. 1503-1512.

12] X. Xu, F. Wang, G. Liu, and F. Qian, "Identification of Hammerstein Systems using Key-Term Separation Principle, Auxiliary Model Improved Particle Swarm Optimization Algorithm, IET Signal Processing vol. 7, no 8, 2013, pp. 766-773.

[13] L. Zhou, X. Li, L. Shan, J. Xia, and W. Chen, "Hierarchical Recursive Least Squares Parameter Estimation of Non-Uniformly Sampled Hammerstein Nonlinear Systems Based on Kalman Filter", Journal of the Franklin Institute vol. 354, no 10, 2017, pp. 4231-4246.

[14] J. Chen, X. Lu, and R. Ding, "Gradient-Based Iterative Algorithm for Wiener Systems with Saturation Dead-Zone Nonlinearities", Journal of Vibration Control vol. 20, no 4, 2014, pp. 634-640.

[15] F. Ding, X. Liu, and M. Liu, "The Recursive Least Squares Identification Algorithm for a Class of Wiener Nonlinear Systems", Journal of the Franklin Institute vol. 353, no 7, 2016, pp. $1518-1526$.

[16] A. Janczak, "Instrumental Variables Approach to Identification of a Class of MIMO Wiener Systems", Nonlinear Dynamics vol. 48, 2007, pp. 275-284.

[17] S. Jing, T. Pan, and Z. Li, "Variable Knot-Based Spline Approximation Recursive Bayesian Algorithm for the Identification of Wiener Systems with Process Noise", Nonlinear Dynamics vol. 90, no. 4, 2017, pp. 2293-2303.

[18] K. Kazlauskas and R. Pupeikis, "On Intelligent Extraction of an Internal Signal in a Wiener System Consisting of a Linear Block Followed by Hard-Nonlinearity", Informatica vol. 24, no 1, 2013, pp. 35-58.

19] J. Kou, W. Zhang, and M. Yin, "Novel Wiener Models with a Time-Delayed Nonlinear Block their Identification", Nonlinear Dynamics vol. 85, no 4, 2016, 2389-2404.

20] L. Li and X. Ren, "Decomposition-based recursive least-squares parameter estimation algorithm for Wiener-Hammerstein systems with dead-zone nonlinearity", International Journal of Systems Science 48, no 11, 2017, pp. 2405-2414.

[21] R. Liu, T. Pan, S. Chen, and Z. Li, "Identification of Non-Uniformly Sampled Wiener Systems with Dead-Zone Non-Linearities", Mathematical Computer Modelling of Dynamical Systems vol. 23, no 6, 2017, pp. 595-612.

22] M. Pawlak, Z. Hasiewicz, and P. Wachel, "On Nonparametric Identification of Wiener systems", IEEE Trans, Signal Processing vol. 55, 2007, pp. 482-492.

[23] P. S. Pal, R. Kar, D. Mandal, and S. P. Ghoshal, "Parametric Identification with Performance Assessment of Wiener Systems Using Brain Storm Optimization Algorithm", Circuits Systems, Signal Processing vol. 36, no. 8, 2017, pp. 3143-3181.

[24] A. Radouane, F. Giri, F. Ikhouane, T. Ahmed-ali, F. Z. Chaoui, and A. Brouri, "System Identification of a Class of Wiener Systems with Hysteretic Nonlinearities", Int. Journal of Adaptive Control Signal Processing vol. 31, no 3, 2017, pp. 332-359.

[25] J. Vörös, "Parameter Identification of Wiener Systems with Multisegment Piecewise-Linear Nonlinearities", Systems Control Letters vol. 56, no 2, 2007, pp. 99-105.

26] P. Wachel and G. Mzyk, "Direct Identification of the Linear Block in Wiener System", Int. Journal of Adaptive Control Signal Processing vol. 30, no 1, 2016, pp. 93-105.

[27] E. W. Bai, "A Blind Approach to the Hammerstein-Wiener Model Identification", Automatica vol. 38, 2002, pp. 967-979.

[28] B. Ni, M. Gilson, and H. Garnier, "Refined Instrumental Variable Method for HammersteinWiener Continuous-Time Model Identification", IET Control Theory Applications vol. 7, no 9, 2013, pp. 1276-1286.

[29] J. Vörös, "An Iterative Method for Hammerstein-Wiener Systems Parameter Identification", Journal of Electrical Engineering vol. 55, no 11-12, 2004, pp. 328-331. 
[30] Y. Wang and F. Ding, "A Filtering Based Multi-Innovation Gradient Estimation Algorithm Performance Analysis for Nonlinear Dynamical Systems", IMA Journal of Applied Mathematics vol. 82, no 6, 2017, pp. 1171-1191.

[31] A. Wills and B. Ninness, "Generalised HammersteinWiener System Estimation a Benchmark Application", Control Engineering Practice vol. 20, no 11, 2012, pp. 1097-1108.

[32] F. Yu, Z. Mao, P. Yuan, D. He, and M. Jia, "Recursive Parameter Estimation for Hammerstein-Wiener Systems using Modified EKF Algorithm", ISA Transactions vol. 70 2017, pp. 104-115.

[33] B. Zhang, H. Hong, and Z. Mao, "Adaptive Control of HammersteinWiener Nonlinear Systems", International Journal of Systems Science vol. 47, no 9, 2016, pp. 2032-2047.

[34] V. Kalaš, L. Jurišica, M. Žalman, S. Almássy, P. Sivicek, A. Varga, and D. Kalaš, "Nonlinear Numerical Servosystems", Bratislava Alfa/SNTL (in Slovak), 1985.

[35] V. Cerone and D. Regruto, "Bounding the Parameters of Linear Systems with Input Backlash", IEEE Trans. Automatic Control vol. 52, no 3, 2007, pp. 531-536.

[36] R. L. Dong and Y. H. Tan, "On-line Identification Algorithm Convergence Analysis for Sandwich Systems with Backlash", International Journal of Control Automation Systems vol. 9, no 3, 2011, pp. 588-594.

[37] F. Giri, Y. Rochdi, F. Z. Chaoui, and A. Brouri, "Identification of Hammerstein Systems in Presence of Hysteresis-Backlash Hysteresis-Relay Nonlinearities", Automatica vol. 44, no 3, 2008, pp. $767-775$.

[38] X. Huang and J. Wang, "Identification of Ground Vehicle Steering System Backlash", Journal of Dynamic Systems Measurement, Control - Transactions of the ASME vol. 135, no 1, 2013, art, no, 011014,

[39] G. Lai, C. Wen, Z. Liu, Y. Zhang, C. L. P. Chen, and S. Xie, "Adaptive Inverse Compensation for Actuator Backlash with Piecewise Time-Varying Parameters", International Journal of Control vol. 91, no 2, 2018, pp. 337-345.

[40] L. Li, X. Ren, and F. Guo, "Modified Multi-Innovation Stochastic Gradient Algorithm for Wiener-Hammerstein Systems with Backlash", Journal of the Franklin Institute vol. 355, no 9, 2018, pp. 4050-4075.

[41] Z. Shi, Y. Wang, and Z. Ji, "A Multi-Innovation Recursive Least Squares Algorithm with a Forgetting Factor for Hammerstein CAR Systems with Backlash", Circuits Systems, Signal Processing vol. 35, no 12, 2016, pp. 4271-4289.

[42] J. Vörös, "Modeling Identification of Systems with Backlash", Automatica vol. 46, no 2, 2010, pp. 369-374.
[43] Z. Zhou, Y. Tan, Y. Xie, and R. Dong, "State Estimation of a Compound Non-Smooth Sandwich System with Backlash Dead Zone", Mechanical Systems Signal Processing vol. 83, no 2017, pp. 439-449.

[44] J. Vörös, "Identification of Nonlinear Dynamic Systems with Input Saturation Output Backlash using Three-Block Cascade Models", Journal of the Franklin Institute vol. 351, no 12, 2014, pp. $5455-5466$.

[45] J. Vörös, "Modelling Identification of Nonlinear Cascade Systems with Backlash Input Static Output Nonlinearities", Mathematical Computer Modelling of Dynamical Systems vol. 24, no 6,2014 , pp. 573-589.

[46] L. Ljung and T. Söderström, "Theory and Practice of Recursive Identification", Massachusetts MIT Press, 1983.

[47] M. Chidambaram, "Computer Control of Processes", New York CRC Press, 2001.

[48] F. Giri, A. Radouane, A. Brouri, and F. Z. Chaoui, "Combined Frequency-Prediction Error Identification Approach for Wiener Systems with Backlash Backlash-Inverse Operators", Automatica vol. 50, 2014, pp. 768-783.

49] J. Reyland and E. W. Bai, "Generalized Wiener System Identification: General Backlash Nonlinearity Finite Impulse Response Linear Part", Int. Journal of Adaptive Control Signal Processing vol. 28, 2014, pp. 1174-1188.

[50] J. Vörös, "Parametric Identification of Systems with General Backlash", Informatica vol. 23, no 2, 2012, pp. 283-298.

[51] J. Vörös, "Modeling Identification of Nonlinear Cascade Sandwich Systems with General Backlash", Journal of Electrical Engineering vol. 65, no 2, 2014, pp. 104-110.

Received 11 January 2019

Jozef Vörös (Ing, $\mathrm{PhD}$ ) was born in Hurbanovo on July 9, 1949. He graduated in automatic control from the Faculty of Electrical Engineering of Slovak Technical University, Bratislava in 1974 and received his PhD degree in control theory from the Institute of Technical Cybernetics of the Slovak Academy of Sciences, Bratislava in 1983. Since 1992 he has been with the Faculty of Electrical Engineering and Information Technology at the Slovak Technical University in Bratislava where he is acting as a senior research scientist in the Institute of Control and Industrial Informatics. His research interests include the analysis and identification of nonlinear systems. He is also interested in the area of mobile robot path planning using quadtree and octree representations. 